\title{
Effects of exercise training with blood flow restriction on vascular function in adults: a systematic review and meta- analysis
}

\author{
Elisio A Pereira-Neto ${ }^{\text {Corresp., } 1,2}$, Hayley Lewthwaite ${ }^{1,2,3}$, Terry Boyle $^{2,4}$, Kylie Johnston ${ }^{1,2}$, Hunter Bennett ${ }^{2,5}$, Marie T \\ Williams $^{1,2}$ \\ ${ }^{1}$ Innovation, IMPlementation And Clinical Translation in Health (IIMPACT), University of South Australia, Adelaide, South Australia, Australia \\ 2 Allied Health and Human Performance, University of South Australia, Adelaide, South Australia, Australia \\ 3 Department of Kinesiology and Physical Education, McGill University, Montreal, Quebec, Canada \\ 4 Australian Centre for Precision Health, University of South Australia, Adelaide, South Australia, Australia \\ 5 Alliance for Research in Exercise, Nutrition, and Activity (ARENA), University of South Australia, Adelaide, South Australia, Australia \\ Corresponding Author: Elisio A Pereira-Neto \\ Email address: elisio.pereira_neto@mymail.unisa.edu.au
}

Background. Blood flow restricted exercise (BFRE) improves physical fitness, with theorized positive effects on vascular function. This systematic review and meta-analysis aimed to report 1 ) the effects of BFRE on vascular function in adults with or without chronic health conditions, and 2) adverse events and adherence reported for BFRE.

Methodology. Five electronic databases were searched by two researchers independently to identify studies reporting vascular outcomes following BFRE in adults with and without chronic conditions. When sufficient data were provided, meta-analysis and exploratory meta-regression were performed. Results. Twenty-six studies were included in the review (total participants $n=472 ; n=41$ older adults with chronic conditions). Meta-analysis ( $k=9$ studies) indicated that compared to exercise without blood flow restriction, resistance training with blood flow restriction resulted in significantly greater effects on endothelial function (SMD 0.76; $95 \% \mathrm{Cl}[0.36,1.14]$ ). No significant differences were estimated for changes in vascular structure (SMD $-0.24 ; 95 \% \mathrm{Cl}[-1.08$, 0.59]). In exploratory meta-regression analyses, several experimental protocol factors (design, exercise modality, exercised limbs, intervention length and number of sets per exercise) were significantly associated with the effect size for endothelial function outcomes. Adverse events in BFRE studies were rarely reported. Conclusion. There is limited evidence, predominantly available in healthy young adults, on the effect of BFRE on vascular function. Signals pointing to effect of specific dynamic resistance exercise protocols with blood flow restriction ( $\geq 4$ weeks with exercises for the upper and lower limbs) on endothelial function warrant further investigation. 


\section{Effects of exercise training with blood flow restriction \\ 2 on vascular function in adults: A systematic review \\ 3 and meta-analysis}

4

Short title: Blood flow restricted exercise and vascular function

6

Elisio Alves Pereira-Neto ${ }^{1,2}$, Hayley Lewthwaite ${ }^{1,2,3}$, Terry Boyle ${ }^{1,4}$, Kylie N Johnston ${ }^{1,2}$, Hunter Bennett $^{1,5}$, Marie T Williams ${ }^{1,2}$

${ }^{1}$ University of South Australia, Allied Health and Human Performance, Adelaide - Australia ${ }^{2}$ University of South Australia, Innovation, IMPlementation And Clinical Translation in Health (IIMPACT), Adelaide - Australia

${ }^{3}$ McGill University, Department of Kinesiology and Physical Education, Montreal - Canada ${ }^{4}$ University of South Australia, Australian Centre for Precision Health, Adelaide - Australia ${ }^{5}$ University of South Australia, Alliance for Research in Exercise, Nutrition, and Activity (ARENA), Adelaide, Australia

Corresponding Author:

Elisio Alves Pereira-Neto ${ }^{1,2}$

University of South Australia, City East Campus, Centenary Building, Level 8 - North Terrace, Adelaide, SA 5000

E-mail address: elisio.pereira_neto@mymail.unisa.edu.au

23

24

25

26

27

28

29

30

31

32

33

34

35

36

37

38

39

40

41 


\section{Abstract}

43 Background. Blood flow restricted exercise (BFRE) improves physical fitness, with theorized 44 positive effects on vascular function. This systematic review and meta-analysis aimed to report

45 1) the effects of BFRE on vascular function in adults with or without chronic health conditions, 46 and 2) adverse events and adherence reported for BFRE.

47 Methodology. Five electronic databases were searched by two researchers independently to 48 identify studies reporting vascular outcomes following BFRE in adults with and without chronic 49 conditions. When sufficient data were provided, meta-analysis and exploratory meta-regression 50 were performed.

51 Results. Twenty-six studies were included in the review (total participants $n=472 ; n=41$ older without blood flow restriction, resistance training with blood flow restriction resulted in significantly greater effects on endothelial function (SMD $0.76 ; 95 \%$ CI $[0.36,1.14]$ ). No significant differences were estimated for changes in vascular structure (SMD -0.24; 95\% CI [$1.08,0.59])$. In exploratory meta-regression analyses, several experimental protocol factors (design, exercise modality, exercised limbs, intervention length and number of sets per exercise) were significantly associated with the effect size for endothelial function outcomes. Adverse events in BFRE studies were rarely reported.

Conclusion. There is limited evidence, predominantly available in healthy young adults, on the effect of BFRE on vascular function. Signals pointing to effect of specific dynamic resistance exercise protocols with blood flow restriction ( $\geq 4$ weeks with exercises for the upper and lower limbs) on endothelial function warrant further investigation.

\section{Introduction}

Blood flow restricted exercise (BFRE) is low-intensity exercise performed with restriction of blood flow to the active muscle group [1]. Improvements in muscle mass and strength, sports performance and aerobic and functional capacity following BFRE have been reported to be similar to higher intensity traditional exercise [2-4]. It has been suggested that BFRE may, therefore, benefit populations that cannot perform traditional exercise at an intensity high enough for maximal physiological adaptations (for example, the elderly or people with chronic obstructive pulmonary disease [COPD] or osteoporosis) [3-6]. However, these populations often have comorbidities related to impaired vascular function (VF) [7,8], which increase the risk of cardiovascular events and mortality $[9,10]$. From this perspective, it is important to understand the potential effects, positive or negative, of BFRE on VF.

Improvements in VF following participation in traditional exercise have been reported in systematic reviews $[8,11]$, with an increase in nitric oxide availability and reduction of oxidant enzymes proposed as underlying mechanisms [12]. The hypoxic conditions created during prolonged exercise with blood flow restriction, and the increased shear stress on the artery walls following release of the cuff, may increase the availability of nitric oxide and vascular endothelial growth factor, stimulating angiogenesis and consequently improving VF [13]. Conversely, it is possible that the accumulation of metabolites under anaerobic conditions during BFRE may trigger the metaboreflex, leading to increases in sympathetic activity and vasoconstriction, and greater total peripheral resistance $[14,15]$. Studies that have investigated the effects of BFRE on VF have reported heterogenous results, with both beneficial and deleterious [16-18] effects reported for VF. 
88

89

90

91

92

93

94

95

96

97

98

99

100

101

102

103

104

105

106

107

108

109

110

111

112

113

114

115

116

117

118

119

120

121

122

123

124

125

126

127

128

129

130

131

132

133

Given this conflicting evidence, this systematic review aimed to explore the effects of BFRE on vascular function in adults with or without chronic health conditions. The specific question posed for this review was "In adults, compared to exercise training without blood flow restriction (nonBFRE), what effect does blood flow restricted exercise training (BFRE) have on vascular function?" Secondarily, we aimed to identify BFRE associated adverse events, adherence and attrition.

\section{Survey methodology}

\section{Search strategy}

The review protocol was registered with the International Prospective Register of Systematic Reviews (PROSPERO, CRD42019147408) and reported according to the Preferred Reporting Items for Systematic reviews and Meta-Analyses (PRISMA) [19]. Five electronic databases (PubMed, Web of Science, Scopus, Medline and EmBase) were searched for studies published from inception to $31^{\text {st }}$ August 2019 and updated on $19^{\text {th }}$ August 2020, with no language restriction imposed. The following combination of terms were used within the search strategy: ("blood flow restriction" OR "blood flow occlusion" OR "vascular occlusion" OR "kaatsu training") AND ("vascular function" OR "endothelial function" OR "arterial stiffness" OR "pulse wave velocity" OR "flow-mediated dilatation" OR "VEGF1" OR "nitric oxide") (See Table S1 for example search strategy). The reference lists of relevant studies identified in the screening process were screened for any additional relevant studies.

\section{Eligibility criteria}

Studies were eligible for inclusion in this review if they: a) were a primary study published as full text in a peer-reviewed journal; b) were a controlled trial with or without randomization (parallel groups, cross-over included), with at least one group including BFRE and, at least one group including non-BFRE (exercise of any form); c) were conducted in adults (18 years and over with or without chronic health conditions); d) applied blood flow restriction (BFR) using an external device to promote external pressure in the proximal part of a limb (e.g., cuff, elastic band); and e) reported at least one outcome related to vascular function (e.g., flow-mediated dilatation [FMD], pulse-wave velocity [PWV], nitric oxide). Studies were excluded if they: a) were single group pre-post studies comparing baseline outcomes with outcomes measured during or post-BFRE without a non-BFRE group comparator; or b) used other forms of hypoxic training such as hyperbaric chamber or high-altitude training as the intervention group.

\section{Selection process}

References identified from database searches were imported to systematic review software (Covidence systematic review software, Veritas Health Innovation, Melbourne, Australia) and exact duplicates removed. Title and abstract of references were initially screened for eligibility by two reviewers independently (EPN and MW). Where studies met eligibility or could not confidently be excluded, full text versions were accessed and screened by two reviewers independently (EPN and HB). Differences between reviewers were resolved by discussion and when consensus could not be reached, a third reviewer (MTW) was consulted. Hand searching of reference lists of included papers was undertaken to identify additional eligible studies.

\section{Data extraction}


134 A data extraction template was developed and piloted a priori. Data extraction was performed by 135 two reviewers independently (EPN and HB). Data extracted from studies included the following 136 domains:

137 a) Publication demographics (publication year, authors, country of data collection);

138 b) Study details (design, sample size, participant age [mean, SD] and gender, health status

139 [healthy or clinical condition]);

140 c) Exercise training regimen for intervention and control groups (protocol duration, exercise

141 intensity, frequency and type, blood flow restriction protocol [intervention group], blood flow

142 restriction pressure [intervention group]);

143 d) Primary outcomes: mean and standard deviation (SD) for vascular function outcomes pre- and 144 post-intervention for the BFRE and non-BFRE groups, which could include but were not limited 145 to: vascular structure (e.g., ankle-brachial index, arterial diameter, pulse-wave velocity),

146 endothelial function (e.g., flow-mediated dilatation, resting blood flow, reactive hyperemia blood 147 flow) and biomarkers (e.g., catalase, vascular endothelial growth factor, nitric oxide). For studies

148 where the results for the primary outcomes were presented in figures, WebPlotDigitizer was used 149 to extract data [20];

150 e) Secondary outcomes: adverse events (any unpleasant or unintended event, sign, symptom 151 reported by authors as related to the intervention [21], adherence (percentage of planned sessions 152 attended [22]) and attrition (proportion of randomized participants analyzed [22]).

\section{Study quality - Appraisal of methodological bias}

The methodological quality of the studies was assessed using the Tool for the Assessment of Study Quality and Reporting in Exercise (TESTEX) scale (validity and reliability intra-class correlation coefficient $>0.91$ [23]). This instrument consists of two sections (study quality and reporting). Standardized instructions guided scoring (minimum of 0 and maximum of 15 points), where higher scores indicated better study quality (0-5 points) and reporting (0-10 points) [22]. Assessment was performed by two reviewers independently (EPN and JP) for all eligible studies. Studies were not excluded based on score for methodological quality as this has been shown to reduce precision and increase bias on meta-analysis results [24,25].

\section{Data management and analysis Descriptive analysis}

Details on study publication and protocol characteristics were tabulated and a narrative synthesis was used to summarize study demographics (author, publication year), design, sample characteristics, exercise training regimens and primary and secondary outcomes. Outcomes for vascular function were grouped under three broad constructs: 'Vascular structure', 'Endothelial function' and 'Biomarkers'. Frequency and percentage were used to present data regarding adverse events, adherence and attrition across studies. When adverse events were reported, the number of participants affected, and verbatim description of adverse events were collated.

\section{Meta-analysis}

A meta-analysis was performed where studies were comparable in design, intervention duration and outcomes for Vascular structure and Endothelial function. Meta-analysis was not performed for the Biomarker construct as measures within this construct represent a diverse range of aspects related to vascular function and it was deemed not appropriate to combine them for meta- 
180

181

182

183

184

185

186

187

188

189

190

191

192

193

194

195

196

197

198

199

200

201

202

203

204

205

206

207

208

209

210

211

212

213

214

215

216

217

218

219

220

221

222

223

224

225

analysis. For studies that reported more than one outcome within the Vascular structure and Endothelial function constructs, PWV (vascular structure) and FMD (endothelial function) were chosen as the outcome of interest as these are considered the gold standard [26]. In the absence of studies reporting PWV or FMD, the outcome with the most rigorous validation according to available best evidence, or the most commonly reported outcome across studies, was chosen. As the Ankle-Brachial index (ABI) has a preferred range of normality rather than a direction for improvement [27], studies reporting $\mathrm{ABI}$ as a measure of vascular structure were not included in the meta-analysis.

The standardized mean difference (SMD) was calculated using the post-intervention outcome mean, standard deviation (SD) and sample size for the BFRE (experimental) and non-BFRE (control) groups. When these metrics were not reported, authors were contacted, or SD was calculated from the standard error reported [28]. If a study had more than one control group (e.g., high- and low-intensity non-BFRE), separate meta-analyses were undertaken (e.g., BFRE versus high-intensity non-BFRE and BFRE versus low-intensity non-BFRE). Heterogeneity between studies was analyzed with $I^{2}$ (with values stablished as $\mathrm{I}^{2}=25 \%$ [low], $\mathrm{I}^{2}=50 \%$ [moderate] and $\mathrm{I} 2=75 \%$ [high]) [29] and Cochran's Q test (significance $\mathrm{p}<0.05$ ). Random-effects meta-analyses were used to compare BFRE and non-BFRE groups due to the different characteristics of the interventions used [30,31]. Meta-analyses were conducted using RevMan (Review Manager Version 5.3, The Cochrane Collaboration, 2014).

\section{Meta-regression}

Meta-regression was used to explore associations between BFRE protocol factors and effect sizes of vascular structure and endothelial function outcomes in the BFRE group of included studies. For studies that reported more than one outcome per construct, the outcome of interest was chosen using the same rationale described above. Predictor variables explored were: a) study design (randomized controlled trial or randomized cross-over trial with controlled trial as reference); b) population group (healthy older adults or clinical population with healthy adults as reference); c) exercise modality (treadmill walking, dynamic resistance training or others with handgrip as reference); d) exercised limbs (upper or lower limbs with both limbs as reference); e) length of intervention ( $\geq 4$ weeks with single session as reference); f) number of sets ( 3,4 or $\geq 5$ with $\leq 2$ as reference); g) cuff width (continuous variable); h) approach to determine restriction pressure (arterial occlusion pressure, systolic blood pressure or limb circumference, with predetermined as reference); i) mean restriction pressure (continuous variable); and methodological quality (TESTEX score, continuous variable). Associations between predictor variables and outcome effect sizes were explored when there were $\geq 10$ studies that reported on an outcome within the construct (vascular structure or endothelial function) [28]. Effect sizes and variance were calculated using pre- and post-intervention mean pre-intervention SD, and pre-post correlation coefficient $(r)$. When these metrics were not reported, we obtained this information by (in order or preference): contacting authors; calculating SD standard error or confidence interval and/or calculating $r$ using the mean and SD of change from pre to post [28]; or using the mean of $r$ from all other studies [28]. Random-effects meta-regression models were conducted separately for each outcome construct. Meta-regression was performed in R Studio 1.2.5 (RStudio Inc., Boston, MA, USA) using the package 'metafor'. A two-sided significance level of $\mathrm{p}<0.05$ was used for all analyses.

Peer] reviewing PDF | (2021:02:57682:1:2:NEW 13 Apr 2021) 


\section{Results}

227 The search identified 1047 references, of which, 26 studies were eligible for inclusion in this 228 review (See Figure S1).

229

230

231

232

233

234

235

236

237

238

239

240

241

242

\section{Study design and population}

The majority of included studies were controlled trials (13 randomized controlled trials [RCT], 10 randomized cross-over trials, two non-randomized controlled trials and a case report; see Table S2). There were 15 (58\%) studies that evaluated BFRE versus one other group, six studies had two comparator groups and five studies had three comparator groups (See Table S2). Across the 26 studies, 472 individuals ( 303 males, 169 females, aged 18 to 91 years) were included. The majority of studies ( $\mathrm{n}=23,88 \%$ ) were in non-clinical populations (total $n=431,284$ males, 147 females, aged 18 to 81 years). Three studies recruited participants with chronic conditions (total $\mathrm{n}=41 ; 19$ males, 22 females; aged 58 to 91 years), with each study representing a different clinical condition (coronary artery disease, hypertension, sarcopenia). Overall, methodological quality was moderate (median 9.5) with scores ranging between 6-13 (Details presented in Table S3).

\section{Comparators}

The most frequent comparators to the active intervention (low-intensity exercise with BFR) were exercise training without BFR at low $(\mathrm{n}=17,65 \%)$ or higher $(\mathrm{n}=8,31 \%)$ exercise intensities. Two studies included four groups (with and without BFR at low- and moderate-intensity exercise). Single studies compared different (i) restriction pressures ( 40 and $80 \%$ of maximum occlusion pressure) or (ii) BFR position (upper and lower limbs) with low and high intensity exercise to low and high intensity exercise without BFR. The study that used neuromuscular electrical stimulation (NMES) compared the same intensity of maximum voluntary contraction (MVC) with and without BFR.

\section{Blood flow restricted exercise protocols Exercise mode}

In the majority of studies, BFR was combined with low-intensity dynamic resistance exercise $(\mathrm{n}=18,69 \%)$. Exercise of the lower limbs was performed in $12(46 \%)$ studies, upper limbs in seven studies (27\%) and both upper and lower limbs in seven studies (27\%). Intervention duration varied across studies from a single exercise session $(n=10,39 \%)$ to training durations of 4 and 16 weeks with two to three exercise sessions per week $(n=14,54 \%)$. Two studies included assessment of a single exercise session preceding an intervention of 4 and 6 weeks (See Table S4).

\section{Exercise intensity}

The approach to prescribe exercise intensity differed according to the type of exercise training (See Table S4). For dynamic resistance exercise, the most common method was the onerepetition maximum strength test ( $1 \mathrm{RM})(\mathrm{n}=15 ; 58 \%$, exercise intensity range 20 to $40 \%$ of 1RM). For isometric resistance exercise $(n=4,15 \%)$ and NMES $(n=1,4 \%)$, training intensity was based on a percentage of maximum voluntary contraction (MVC; intensity range between 10 to $60 \% \mathrm{MVC}$ ).

\section{Blood flow restriction (BFR)}


272 All studies used cuffs to achieve BFR (pneumatic or hand-pumped) and, where reported ( $\mathrm{n}=18$, $27369 \%$ ), cuff width ranged between 3 to $23 \mathrm{~cm}$. The selection of restriction pressure was based on a 274 variety of criteria: predetermined non-individualized pressure $(n=14,54 \%)$, percentage of

275 systolic blood pressure (SBP) $(n=8,31 \%)$, percentage of limb occlusion pressure (LOP) $(n=2$, $2768 \%)$, limb circumference $(n=1,4 \%)$ and one study used both SBP and LOP criteria depending on 277 278

279

280

281

282

283

284

285

286

287

288

289

290

291

292

293

294

295

296

297

298

299

300

301

302

303

304

305

306

307

308

309

310

311

312

313

314

315

316

317

318

\section{Primary outcomes}

Table 1 presents a summary of outcomes for Vascular structure, Endothelial function and Biomarkers.

\section{Table 1:}

Summary of vascular function outcomes assessed in the studies and differences between BFR and non-BF $R$ conditions

\section{INSERT TABLE 1}

BFR, Blood flow restriction; HI, High intensity; MI, Moderate intensity; LI, Low-intensity; ABI, Anklebrachial index; PWV, Pulse wave velocity; CAVI, Cardio-ankle vascular index; FMD, Flow-mediated dilatation; RBF, Resting blood flow; RHBF, Reactive hyperemia blood flow; RHI, Reactive hyperemia index; $\mathrm{TpO}_{2}$, Tissue oxygenation; $\mathrm{vWF}$, von Willebrand factor; $\mathrm{VEGF}_{1}$, Vascular endothelial growth factor; NO, Nitric oxide; GH, Growth hormone, FDP, Fibrin degradation products; SOD, Superoxide dismutase; CAT, Catalase; $=$, No statistically significant difference between conditions with and without BFR; (+), BFR condition promoted significant positive changes when compared to non-BFR; (-), BFR condition promoted significant negative changes when compared to non-BFR.

Vascular structure: Across 15 studies, six different outcomes were reported for vascular structure, with vascular compliance being the most frequently reported $(n=6,23 \%)$. Of the 15 studies reporting vascular structure outcomes, 13 (87\%) reported no differences between exercise with and without BFR (Table 1). Significant between group differences in vascular structure were reported in two studies following an exercise intervention of 16 weeks of BFR with resistance training (compared to high-intensity non-BFRE) [32] or a single session of wholebody vibration training with BFR (compared to whole-body vibration without BFR) [16].

Endothelial function: Fourteen studies reported six different outcomes for endothelial function (FMD most frequently reported, $n=8,31 \%$ ). Of these 14 studies, eight (57\%) reported no significant difference in endothelial function outcomes between exercise with and without BFR. For FMD, compared to non-BFRE, improvements were reported following four weeks of BFRE [33], a single session of BFRE (walking) [34] and two weeks of BFRE using handgrip training [17]. Blood flow (resting or reactive hyperemia) was reported to be improved after BFRE, compared to low-intensity non-BFRE, in healthy younger [35] and older adults [36,37] and a single subject with sarcopenia [38]. In studies reporting tissue oxygenation [37,39] or dilatory capacity [40], no significant differences were reported between exercise with and without BFR.

Biomarkers: Ten studies reported seven different biomarker outcomes, with nitric oxide (NO) being the most frequently reported $(n=4,15 \%)$. Of these 10 studies, six $(60 \%)$ studies reported no difference in biomarker outcomes between exercise with and without BFR. Growth hormone $(n=2)$ was increased in the BFRE condition compared to low intensity non-BFRE in healthy adults following a single session of handgrip training [41] and in older individuals after four 
weeks of dynamic resistance exercise [37]. Superoxide dismutase $[42,43](n=2,8 \%)$ and catalase [43] $(\mathrm{n}=1,4 \%)$ showed higher concentrations in the BFRE condition following a single session of treadmill walking in hypertensive older women [42] and in healthy adults after dynamic resistance exercise [43].

\section{Meta-analysis}

Nine studies were sufficiently homogenous (design, training protocol and population) for inclusion in the meta-analysis [32,35-37,44-48]. All studies included in the meta-analysis were in non-clinical populations and explored the effects of BFR with isotonic dynamic resistance training for four weeks or more. Separate models were run comparing the effects of low intensity BFRE to high intensity non-BFRE on vascular structure, and low intensity BFRE to low intensity non-BFRE on endothelial function.

\section{Low intensity BFRE versus high intensity non-BFRE - Vascular structure}

Four studies reported outcomes for vascular structure (vascular compliance [n=3], [32,45,46]; and PWV [n=1] [44]) after four or more weeks of dynamic resistance training (low-intensity BFRE versus high-intensity non-BFRE) (Figure 1). No significant difference was observed between low intensity BFRE and high intensity non-BFRE (SMD -0.24, 95\% CI [-1.08, 0.59], $\mathrm{p}=0.57)$ (Figure 1). Moderate and significant heterogeneity was observed $\left(I^{2}=73 \%, \mathrm{p}=0.01\right)$.

Figure 1: Forest plot presenting comparison of vascular structure between high intensity non-BFR training and low intensity BFRE.

\section{INSERT FIGURE 1}

*Values for PWV were reversed to meet direction of vascular compliance where increase means improvement; Squares represent individual study Hedges' $g$ with size corresponding to weight of study and lines are 95\% confidence intervals (CI); Diamond indicates overall Hedges' $\mathrm{g}$ with its width corresponding to $95 \% \mathrm{CI}$.

\section{Low intensity BFRE versus low intensity non-BFRE - Endothelial function}

Five studies provided sufficient data for meta-analysis of the effect of four or more weeks of low intensity dynamic resistance exercise training with and without BFR on endothelial function (Figure 2). Specific endothelial outcomes were FMD $(n=2)[47,48]$, reactive hyperemia blood flow $(n=2)[35,36]$ and reactive hyperemia index $(n=1)$ [37]. Meta-analysis estimated lowintensity dynamic resistance training with BFR had a significant positive difference compared to low intensity exercise without BFR (SMD 0.76, 95\% CI [0.36, 1.14], $\mathrm{p}<0.001$ ) for endothelial function (Figure 2). Heterogeneity was considered low $\left(I^{2}=7 \%\right)$ and not significant $(\mathrm{p}=0.36)$.

Figure 2: Forest plot presenting comparison of endothelial function between low intensity non-BFR training and low intensity BFRE.

\section{INSERT FIGURE 2}

*Increase means improvement for all outcomes; Squares represent individual study Hedges' $\mathrm{g}$ with size corresponding to weight of study and lines are $95 \%$ confidence intervals (CI); Diamond indicates overall Hedges' $\mathrm{g}$ with its width corresponding to $95 \% \mathrm{CI}$.

\section{Meta-regression}


364 Findings from meta-regression analyses are summarized in Supplemental Table S5 (Lopes et al. 3652019 [38] excluded as case study). For vascular structure, 15 studies were included within meta366 regression models reflecting four vascular structure outcomes: pulse-wave velocity (PWV) $(\mathrm{n}=5)$ $367[34,44,49-51]$, vascular compliance $(n=5)[16,32,45,46,52]$, cardio-ankle vascular index $(n=3)$ $368[47,48,53]$ and arterial diameter $(n=2)[17,33]$. No significant associations were identified

369

370

371

372

373

374

375

376

377

378

379

380

381

382

383

384

385

386

387

388

389

390

391

392

393

394

395

396

397

398

399

400

401

402

403

404

405

406

407

408

409 between BFRE protocol factors and effect sizes (all p-values $>0.05$ ).

For endothelial function, 13 studies were included in the meta-regression reflecting three outcomes: FMD $(n=8)[17,33,40,47-49,54,55]$, reactive hyperemia blood flow $(n=4)$ $[35,36,49,50]$ and reactive hyperemia index $(n=1)$ [37]. Study design was significantly associated with effect size, with randomized cross-over trials associated with smaller effect sizes compared to control trials (regression estimate $[\beta]$ of $-2.91 ; 95 \%$ CI $[-5.40,-0.42] ; p=0.02$ ). For exercise modality, compared to handgrip training, treadmill walking $(\beta=-2.62 ; 95 \%$ CI $[-4.18,-1.06)]$; $\mathrm{p}=0.001)$ and dynamic resistance training $(\beta=1.64 ; 95 \% \mathrm{CI}[0.83,2.45] ; \mathrm{p}<0.001)$ were associated with significantly smaller and larger effects on endothelial function, respectively. Upper limb only exercise was associated with smaller effect sizes ( $\beta=-2.22$; 95\% CI [-4.23, $0.19] ; p=0.03$ ) compared to exercise of the lower and upper limbs combined. For the number of exercise sets, compared to studies of $\leq 2$ sets, exercise protocols with three $(\beta=1.66 ; 95 \% \mathrm{CI}$ $[0.21,3.11] ; p=0.02)$ and four $(\beta=1.54 ; 95 \%$ CI $[0.03,3.05] ; p=0.05)$ sets were associated with larger effect sizes, while protocols with five or more sets $(\beta=2.5 ; 95 \%$ CI $[-4.69,-0.30])$; $\mathrm{p}=0.03$ ) were associated with smaller effect sizes. Meta-regression analysis for length of intervention was performed using 'single session' studies as the reference for duration, with durations explored as a continuous variable (number of weeks, $\mathrm{p}>0.05$ ) or categorical variable of $\geq 4$ weeks (e.g., all studies with 4 weeks or more grouped in one category). Compared to a single session, interventions of $\geq 4$ weeks were associated with larger effect sizes $(\beta=1.92 ; 95 \% \mathrm{CI}$ $[0.39,3.46] ; \mathrm{p}=0.01)$.

\section{Secondary outcomes}

Supplemental Table S6 presents a summary of adverse events, adherence, and attrition rates. The majority of studies $(n=18,69 \%)$ did not report the presence or absence of adverse events. Seven studies explicitly reported an absence of adverse events $(n=7,27 \%)$. A single study reported an initial sensation of leg numbness in two participants once they commenced BFRE, which did not persist beyond the first minute of exercise [42] (2 out of 16 hypertensive older women). Adherence and attrition were reported in $11(42 \%)$ and six $(23 \%)$ studies, respectively. Adherence was reported or calculated as $100 \%$ for 11 studies and dropout rates ranged from 5 to $40 \%$.

\section{Discussion}

This systematic review aimed to report BFRE effects on vascular function in adults with or without chronic health conditions and, secondarily, to identify BFRE associated adverse events, adherence and attrition. To the best of our knowledge, this systematic review presents the first quantitative analyses exploring differences between BFRE and non-BFRE on vascular function, and the first exploratory meta-regression model to explore the influence of BFRE protocol components on vascular function. We found that the body of evidence is modest, heterogenous and predominantly included apparently healthy participants. With these caveats in mind, the main findings of this review were: a) a variety of outcomes were used to assess vascular function in the domains of 
410 vascular structure, endothelial function and biomarkers; b) compared to non-BFRE, meta-analysis 411 favored dynamic resistance exercise with BFR for endothelial function outcomes (no difference 412 between interventions for vascular structure outcomes); c) study design, mode of exercise, training 413 volume and length of exercise intervention were predictors of endothelial function effect size with 414 415

416 Previously, BFRE research has focused on muscle strength and size [56] and aerobic capacity 417 [57], with rising interest in other aspects such as blood pressure [5] and perceptual measures 418 [58]. This review suggests that the body of evidence for BFRE and VF is small, with heterogeneous exercise protocols and predominantly in healthy individuals. Interest is increasing concerning potential use of BFRE within older clinical populations (e.g., cardiovascular disease and chronic lung conditions) [3]. There appears to be little information available concerning efficacy and safety of BFRE and impact upon VF specific to people living with chronic conditions - a clinical population where VF is likely impaired. We considered VF as a number of aspects related to vessel structure, functioning of the endothelium and substances which regulate both structure and functioning. This approach was required as few studies reported use of the same outcome measure (e.g., FMD was the most common outcome but reported by approximately one third of studies), which limited our ability to perform more robust analysis [28].

\section{BFRE and Vascular structure}

Our meta-analysis did not identify significant differences between BFRE and non-BFRE for arterial stiffness outcomes. Increased sympathetic nervous system activity is a common concern with BFRE [14]. The reduction of blood flow to the muscle increases vascular peripheral resistance and metabolite concentration, which may trigger baro- and chemoreceptors that stimulate sympathetic outflow [33]. Domingos and Polito (2018)'s meta-analysis reported BFRE to cause greater increases in blood pressure than low intensity traditional resistance training [5], indicating exercise-induced increases in sympathetic activity, which might be expected to increase arterial stiffness and negatively affect vascular structure. The same meta-analysis [5] and previous randomized trials $[59,60]$ have reported a more pronounced post-exercise hypotensive effect for BFRE compared to high- and low intensity traditional resistance training, which is indicative of reduced sympathetic activity [15]. Despite greater sympathetic activity with BFRE compared to low intensity exercise without BFR, the post-exercise reduction of sympathetic outflow associated with BFRE could potentially act as a protector of the vascular system.

\section{BFRE and Endothelial Function}

In healthy individuals, our meta-analysis indicated that four or more weeks of low intensity dynamic resistance exercise with BFR was associated with greater improvements in endothelial function compared to low intensity non-BFRE. Increased shear stress is hypothesized to be a modulator of chronic endothelial function changes following exercise [8,17]. Improvements in FMD following traditional moderate-to-high dynamic resistance exercise have been proposed to be associated with the process of intermittent blood flow in the small muscular arteries resulting from muscle contraction, increasing shear stress in the artery walls and promoting release of vasodilator factors (i.e., NO, VEGF, GH) [17]. It is possible that hypoxic conditions created during prolonged exercise with blood flow occlusion and/or the increased shear stress following 
456 release of the cuff, increase the availability of vasodilator factors consequently improving

457 endothelial function.

458

459

460

461

462

463

464

465

466

467

468

469

470

471

472

473

474

475

476

477

478

479

480

481

482

483

484

485

486

487

488

489

490

491

492

493

494

495

496

497

498

499

500

501

\section{BFRE and Vascular Function Biomarkers}

Few studies included in this review $(n=10 ; 35 \%)$ reported VF biomarker outcomes $[32,37,39,41-$ $43,47,48,53,54]$. Within these studies, seven different outcomes were reported, which in included outcomes not unique to vascular function (i.e., growth hormone [41]). Only four of these studies reported between group differences for VF biomarkers [37,41-43] and, while all four favored BFRE, these studies differed in design, restriction pressure, exercise regimen, exercise intensity and assessed different outcomes (growth hormone, von Willebrand factor, catalase and superoxide dismutase) making it difficult to draw conclusions on the overall effects of BFRE on vascular function biomarkers. Given the variety of outcomes observed in this review, and the heterogeneity of study designs/protocols, it is not clear which biomarkers should be considered as priorities for studies investigating BFRE and vascular function.

\section{Which characteristics of BFRE protocols were associated with vascular function or structure effects sizes? Findings of the meta-regression}

Our meta-regression results should be interpreted with caution due to the small number of studies (total $\mathrm{k}=15$ for vascular structure and $\mathrm{k}=13$ for endothelial function, with fewer common studies for each specific outcome within the broader domains), high heterogeneity between study protocols, and recognition that within each bivariate model, effect sizes for each variable and differences between strength of association are compared with a reference variable, negating identification of the causal influence of different predictors [28]. In addition, only four (16\%) studies reported a sample size estimate, making it difficult to determine whether the remaining studies $(n=22 ; 84 \%)$ were adequately powered. Underpowered studies may provide inflated effect sizes and reduce the chance of detecting a true difference [61,62]. With these forewarnings in mind, a number of protocol 'signals' were evident for endothelial function effects (but not vascular structure).

Intervention duration as a categorical, but not a continuous variable, was a significant predictor of effect size. Endothelial function measures are prone to change after a single bout of exercise [12], the degree of which appears to differ between exercise type [12]. As such, the time frame required for physiological changes to endothelial function in response to chronic exercise varies. It is likely that other BFRE protocol factors (e.g., approach to determine pressure, cuff width) influence the relationship between intervention duration and endothelial function, which could only be detected in a multivariate analysis.

Consistent with known exercise training principles [63], we found exercise volume and mode (engaging larger muscle groups) to be important for endothelial function outcomes. Previous meta-analysis on non-BFRE (i.e., traditional exercise training) identified higher exercise volumes, compared to lower exercise volumes, resulted in greater improvements in skeletal muscle hypertrophy [64], potentially caused by an increased release of neurohumoral factors (i.e., GH, nitric oxide, vascular endothelial growth factor). These same neurohumoral factors are known to modulate endothelial function [63]. It is, therefore, possible that higher training volumes will also impact vascular outcomes. Within our meta-regression, there were also variables signaling potential harm - albeit from a single study. The association between training 
502 at five or greater sets per exercise and smaller effect sizes for endothelial function may reflect the 503 concept that elevated training volumes can produce higher inflammatory responses [65] and, 504 consequently, decrease endothelial function. This is in agreement with a recent review of BFRE 505 methodology, application and safety which recommended BFR training use dynamic resistance 506 exercise of four sets per exercise [1].

507

508

509

510

511

512

513

514

515

516

517

518

519

520

521

522

523

524

525

526

527

528

529

530

531

532

533

534

535

536

537

538

539

540

541

542

543

544

545

546

Within our review, reported cuff widths ranged between 3 to $23 \mathrm{~cm}(\mathrm{n}=18)$ and the most common approach reported for determining restriction pressure was use of a predetermined, nonindividualized pressure $(n=13)$ with systolic blood pressure $(n=10)$ and arterial occlusion pressure $(n=3)$ accounting for the remaining approaches. The approach used to determine restriction pressure (e.g., predetermined non-individualized, systolic blood pressure or LOP) did not significantly predict of effect size for arterial structure or endothelial function. The review published by Patterson et al. (2019) recommended using a percentage of an individual's LOP [1]. The recency of this recommendation (published May 2019) is likely to explain why the majority $(92 \%)$ of studies did not use LOP to prescribe restriction pressure. Limitations of alternative approaches to determining BFRE restriction training pressures include inter limb/individual variation in limb circumferences necessitating different cuff pressures and inconsistent associations between blood pressure and the pressure required to achieve occlusion [66]. Higher restriction pressures (i.e., non-individualized $220 \mathrm{mmHg}$, which is $100 \mathrm{mmHg}$ higher than normal SBP) also appear to increase discomfort and cardiovascular responses [67], which may increase the risk of adverse vascular events [66]. Future studies in BFRE and vascular function are more likely to individually prescribe training pressure using a percentage of LOP.

Blood pressure responses following BFRE have been explored in prior meta-analysis (23 studies included in the analysis of normotensive and hypertensive individuals [5]), with hypertensive participants showing higher blood pressure changes. There is very little data available on the effects of BFRE in the vascular function of older individuals with chronic conditions. Our review included 41 individuals (of the total $n=472$ ) with chronic conditions, and the review by Domingos and Polito (2018) included 95 (of the total $n=325$ ). As populations living with chronic conditions often present with increased arterial stiffness and endothelial dysfunction [10], it is likely that they will have different vascular function responses to BFRE than healthy adults. Notably, the majority of studies included within this review excluded participants with comorbidities such as peripheral vascular disease (assessed by the ankle-brachial index) [66]. The findings of this review concerning the effects of BFRE on vascular function should therefore not be generalized to older populations living with chronic cardiovascular comorbidities.

\section{Secondary outcomes}

In a survey [67] of 115 practitioners who used BFRE, 39\% $(n=60)$ reported at least one side effect observed in their practice. Whereas in Hughes et al. (2017)'s review of BFRE [3] and our review, $50 \%$ and $69 \%$ of studies (respectively) made no mention of adverse events. In these studies, it is not clear if adverse event did not occur or if they were not assessed or reported. The potential underreporting of either presence or absence of adverse events makes it difficult to determine the safety of BFRE and transparent reporting should be considered a priority in future BFRE studies. 
547 Strengths of this review include a comprehensive search of major databases related to the review 548 topic, with no language or time restrictions, and a rigorous approach to conducting the review 549 and reporting of results. Due to heterogeneity of exercise regimens and outcomes in the studies 550 included, it was not possible to perform adjustment for potential confounding factors in the meta-

551

552

553

554

555

556

557

558

559

560

561

562

563

564

565

566

567

568

569

570

571

572

573

574

575

576

577

578

579

580

581

582

583

584

585

586

587

588

589 regression. Publication bias was not assessed as the funnel plot tests to detect asymmetry are compromised in meta-analysis with small numbers $(<10)$ of studies [28]. Translation of our results to clinical populations is limited as the majority of included studies were in healthy individuals.

\section{Conclusions}

BFRE has been suggested as potentially useful in older clinical populations unable to train at the intensity and duration essential for physiological responses. However, such populations may also have altered vascular function. This review identified a small, heterogeneous evidence base for the effect of BFRE on vascular function/structure with studies prominently including younger healthy individuals. Meta-analysis findings suggested BFRE training to have a positive effect compared to non-BFRE on endothelial function, but not on vascular structure. There are indications that BFRE protocol factors, such as the use of dynamic resistance training, $\geq 4$ weeks duration and exercise protocols which include training for both upper and lower limbs, are associated with larger effect sizes for vascular function outcomes.

For clinicians/researchers planning future studies of BFRE, consideration should be given to recruiting older people with and without chronic conditions, including outcomes for vascular function, adverse events and tolerability and adopting recommendations from the review by Patterson et al. (2019) [1] for determining restriction pressure and prescribing exercise, as this will standardize protocols and facilitate comparison of findings.

\section{Key findings:}

- The evidence regarding the effects of blood flow restricted exercise (BFRE) training in vascular function is small and heterogeneous.

- Our results suggest that BFRE training may have a positive effect on endothelial function and that BFRE protocol factors (e.g., mode and duration of exercise) are associated with larger effect sizes for vascular function outcomes.

- It is necessary to report adverse events and include vascular function outcomes in future BFRE training studies to clarity its safety.

\section{References}

1. Patterson SD, Hughes L, Warmington S, Burr J, Scott BR, Owens J, Abe T, Nielsen JL, Libardi CA, Laurentino G, Neto GR, Brandner C, Martin-Hernandez J, Loenneke J. Blood flow restriction exercise: Considerations of methodology, application, and safety. Front Physiol 2019; 10:1-15.

2. Bennett H, Slattery F. Effects of Blood Flow Restriction Training on Aerobic Capacity and Performance. J Strength Cond Res 2018; 0:1-12. 
590 3. Hughes L, Paton B, Rosenblatt B, Gissane C, Patterson SD. Blood flow restriction training in 591 clinical musculoskeletal rehabilitation: a systematic review and meta-analysis. Br J Sports Med 592 2017; 51:1003-11.

593 4. Pereira Neto EA, Bittar ST, Silva J, Pfeiffer P, Santos H, Cirilo-Sousa M. Walking with blood 594 flow restriction improves the dynamic strength of women with osteoporosis. Brazilian J Sport 595 Med 2018; 24:135-9.

596 5. Domingos E, Polito MD. Blood pressure response between resistance exercise with and 597 without blood flow restriction: A systematic review and meta-analysis. Life Sci 2018; 209:12259831.

599 6. Patterson SD, Hughes L, Head P, Warmington S, Brandner C. Blood flow restriction training: 600 A novel approach to augment clinical rehabilitation: How to do it. Br J Sports Med 2017; 601 51:1648-9.

602 7. Yoo J-I, Kim M-J, Na J-B, Chun Y-H, Park Y-J, Park Y, Hah Y-S, Ha Y-C, Park KS.

603 Relationship between endothelial function and skeletal muscle strength in community dwelling 604 elderly women. J Cachexia Sarcopenia Muscle 2018; 9:1034-41.

605 8. Pedralli ML, Eibel B, Waclawovsky G, Schaun MI, Nisa-Castro-Neto W, Umpierre D, 606 Pescatello H, Tanaka H, Lehnen AM. Effects of exercise training on endothelial function in 607 individuals with hypertension: a systematic review with meta-analysis. J Am Soc Hypertens 608 2018; 12:e65-75.

609 9. Ambrosino P, Lupoli R, Iervolino S, De Felice A, Pappone N, Storino A, Minno MNDD. 610 Clinical assessment of endothelial function in patients with chronic obstructive pulmonary 611 disease: a systematic review with meta-analysis. Intern Emerg Med 2017; 12:877-85.

612 10. Chen W, Thomas J, Sadatsafavi M, FitzGerald JM. Risk of cardiovascular comorbidity in 613 patients with chronic obstructive pulmonary disease: A systematic review and meta-analysis.

614 Lancet Respir Med 2015; 3:631-9.

615 11. Qiu S, Cai X, Yin H, Sun Z, Zügel M, Steinacker JM, Schuman U. Exercise training and 616 endothelial function in patients with type 2 diabetes: a meta-analysis. Cardiovasc Diabetol 2018; 617 17:64.

618 12. Ashor AW, Lara J, Siervo M, Celis-Morales C, Oggioni C, Jakovljevic DG, Mathers JC.

619 Exercise Modalities and Endothelial Function: A Systematic Review and Dose-Response Meta620 Analysis of Randomized Controlled Trials. Sport Med 2015; 279-96.

621 13. Horiuchi M, Okita K. Blood flow restricted exercise and vascular function. Int J Vasc Med

622 2012; 17.

623 14. da Cunha Nascimento D, Schoenfeld BJ, Prestes J. Potential Implications of Blood Flow

624 Restriction Exercise on Vascular Health: A Brief Review. Sport Med 2020; 50:73-81.

625 15. Cristina-Oliveira M, Meireles K, Spranger MD, O’Leary DS, Roschel H, Peçanha T. Clinical

626

627

628

629 16. Karabulut U, Karabulut M, James EG. Small arteries stay stiff for a longer period following 630 vibration exercises in combination with blood flow restriction. Clin Physiol Funct Imaging $6312018 ; 38: 1000-7$.

632 17. Paiva FM, Vianna LC, Fernandes IA, Nobrega AC, Lima RM. Effects of disturbed blood 633 flow during exercise on endothelial function: a time course analysis. Brazilian J Med Biol Res 634 2016; 49.

635 18. Millis RM, Bond V, Curry BH, Kumar K, Pemminati S, Gorantla VR, Kadur K, Millis RM. 
636 Restricted Blood Flow Exercise in Sedentary, Overweight African-American Females May

637 Increase Muscle Strength and Decrease Endothelial Function and Vascular Autoregulation. $J$

638 Pharmacopuncture 2017; 20:23-8.

639 19. Moher D. Preferred reporting items for systematic review and meta-analysis protocols

640 (PRISMA-P) 2015 statement. Syst Rev 2015; 207:1-9.

641 20. Rohatgi A. WebPlotDigitizer . San Francisco, California, USA; 2019.

642 21. Australian National Health and Medical Research C. Australian Code for the Responsible

643 Conduct of Research. 2018.

644 22. Sohanpal R, Hooper R, Hames R, Priebe S, Taylor S. Reporting participation rates in studies

645 of non-pharmacological interventions for patients with chronic obstructive pulmonary disease: A

646 systematic review. Syst Rev 2012; 1:1-13.

647 23. Smart NA, Waldron M, Ismail H, Giallauria F, Vigorito C, Cornelissen V, Dieber G.

648 Validation of a new tool for the assessment of study quality and reporting in exercise training

649 studies: TESTEX. Int J Evid Based Healthc 2015; 13:9-18.

650 24. Stone J, Gurunathan U, Glass K, Munn Z, Tugwell P, Doi SAR. Stratification by quality-

651 induced selection bias in a meta-analysis of clinical trials. J Clin Epidemiol 2019; 107:51-9.

652 25. Higgins JPT, Altman DG, Gøtzsche PC, Jüni P, Moher D, Oxman AD, Schulz KF, Weeks L,

653 Sterne JAC. The Cochrane Collaboration's tool for assessing risk of bias in randomised trials.

654 BMJ 2011; 343:1-9.

655 26. Tousoulis D, Antoniades C, Stefanadis C. Evaluating endothelial function in humans: A

656 guide to invasive and non-invasive techniques. Heart 2005; 91:553-8.

657 27. Resnick H, Lindsay R, Mcdermott M, Devereux R, Jones K, Fabsitz R, Howard BV.

658 Relationship of high and low ankle brachial index to all-cause and cardiovascular disease

659 mortality. Circulation 2004; 109:733-9.

660 28. Higgins J, Thomas J, Chandler J, Cumpston M, Li T, Page M, editors. Cochrane Handbook

661 for Systematic Reviews of Interventions version 6.0 (updated July 2019) . Cochrane; 2019.

662 29. Grant J, Hunter A. Measuring inconsistency in knowledgebases. J Intell Inf Syst 2006;

663 27:159-84.

664 30. Rodrigues CL, Ziegelmann PK. Metanálise: um guia prático. Rev HCPA 2010; 30:436-47.

665 31. Borenstein M, Hedges L V., Higgins JPT, Rothstein HR. A basic introduction to fixed-effect 666 and random-effects models for meta-analysis. Res Synth Methods 2010; 1:97-111.

667 32. Ozaki H, Yasuda T, Ogasawara R, Sakamaki-Sunaga M, Naito H, Abe T. Effects of high-

668 intensity and blood flow-restricted low-intensity resistance training on carotid arterial

669 compliance: Role of blood pressure during training sessions. Eur J Appl Physiol 2013; 113:167-

67074.

671 33. Renzi C, Tanaka H, Sugawara J. Effects of Leg Blood Flow Restriction during Walking on

672 Cardiovascular Function. Med Sci Sport Exerc 2010; 42:726-32.

673 34. Credeur DP, Hollis BC, Welsch MA. Effects of Handgrip Training with Venous Restriction

674 on Brachial Artery Vasodilation. Med Sci Sport Exerc 2010; 42:1296-302.

675 35. Patterson SD, Ferguson RA. Increase in calf post-occlusive blood flow and strength

676 following short-term resistance exercise training with blood flow restriction in young women.

677 Eur J Appl Physiol 2010; 108:1025-33.

678 36. Patterson SD, Ferguson RA. Enhancing strength and postocclusive calf blood flow in older

679 people with training with blood-flow restriction. J Aging Phys Act 2011; 19:201-13.

680 37. Shimizu R, Hotta K, Yamamoto S, Matsumoto T, Kamiya K, Kato M, Hamazaki N,

681 Kamekawa D, Akiyama A, Kamada Y, Tanaka S, Masuda T. Low-intensity resistance training

Peerf reviewing PDF | (2021:02:57682:1:2:NEW 13 Apr 2021) 
682 with blood flow restriction improves vascular endothelial function and peripheral blood

683 circulation in healthy elderly people. Eur J Appl Physiol 2016; 116:749-57.

684 38. Lopes KG, Bottino DA, Farinatti P, de Souza M das G, Maranhao PA, de Araujo CM,

685 Bouskela E, Lourenco RA, de Oliveira RB. Strength training with blood flow restriction - a

686 novel therapeutic approach for older adults with sarcopenia? A case report. Clin Interv Aging

$6872019 ; 14: 1461-9$.

688 39. Larkin KA, Macneil RG, Dirain M, Sandesara B, Manini TM, Buford TW. Blood Flow

689 Restriction Enhances Post-Resistance Exercise Angiogenic Gene Expression. Med Sci Sport

690 Exerc 2012; 44:2077-83.

691 40. Hunt JEA, Walton LA, Ferguson RA. Brachial artery modifications to blood flow-restricted

692 handgrip training and detraining. J Appl Physiol 2012; 112:956-61.

693 41. Natsume T, Ozaki H, Saito AI, Naito H. Neuromuscular electrical stimulation with blood

694 flow restriction increases serum growth hormone concentration. Gazz Medica Ital Arch per le Sci

695 Mediche 2018; 177:599-605.

696 42. Barili A, Corralo V da S, Cardoso AM, Mânica A, Bonadiman B da SR, Bagatini MD, da

697 Silva-Griogoletto ME, de Oliveira GA, de Sa CA. Acute responses of hemodynamic and

698 oxidative stress parameters to aerobic exercise with blood flow restriction in hypertensive elderly

699 women. Mol Biol Rep 2018; 45:1099-109.

700 43. Boeno FP, Ramis TR, Farinha JB, de Lemos LS, Medeiros N da S, Ribeiro JL. Acute effects

701 of strength exercise with blood flow restriction on vascular function of young healthy males. $J$

702 Vasc Bras 2018; 17:122-7.

703 44. Clark BC, Manini TM, Hoffman RL, Williams PS, Guiler MK, Knutson MJ, McGlynn ML,

704 Kushnick MR. Relative safety of 4 weeks of blood flow-restricted resistance exercise in young,

705 healthy adults. Scand J Med Sci Sports 2011; 21:653-62.

706 45. Fahs CA, Rossow LM, Loenneke JP, Thiebaud RS, Kim D, Bemben DA, Bemben MG.

707 Effect of different types of lower body resistance training on arterial compliance and calf blood

708 flow. Clin Physiol Funct Imaging 2012; 32:45-51.

709 46. Mouser JG, Mattocks KT, Buckner SL, Dankel SJ, Jessee MB, Bell ZW, Abe T, Bentley JP, 710 Loenneke JP. High-pressure blood flow restriction with very low load resistance training results

711 in peripheral vascular adaptations similar to heavy resistance training. Physiol Meas 2019; 40.

712 47. Yasuda T, Fukumura K, Iida H, Nakajima T. Effects of detraining after blood flow-restricted

713 low-load elastic band training on muscle size and arterial stiffness in older women. Springerplus

$7142015 ; 4$.

715 48. Yasuda T, Fukumura K, Uchida Y, Koshi H, Iida H, Masamune K, Yamasoba T, Sato Y,

716 Nakajima T. Effects of Low-Load, Elastic Band Resistance Training Combined With Blood

717 Flow Restriction on Muscle Size and Arterial Stiffness in Older Adults. J Gerontol Ser A-

718 BIOLOGICAL Sci Med Sci 2015; 70:950-8.

719 49. Credeur DP, Jones R, Stanford D, Stoner L, McCoy S, Jessee M. Central cardiovascular 720 hemodynamic response to unilateral handgrip exercise with blood flow restriction. Eur J Appl

721 Physiol 2019; 119:2255-63.

722 50. Fahs CA, Rossow LM, Thiebaud RS, Loenneke JP, Kim D, Abe T, Beck TW, Feeback DL,

723 Bemben DA, Bemben MG. Vascular adaptations to low-load resistance training with and without

724 blood flow restriction. Eur J Appl Physiol 2014; 114:715-24.

725 51. Figueroa A, Vicil F. Post-exercise aortic hemodynamic responses to low-intensity resistance

726 exercise with and without vascular occlusion. Scand J Med Sci Sports 2011; 21:431-6.

727 52. Sardeli AV, Santos L do C, Venturini Ferreira ML, Gaspari AF, Rodrigues B, Cavaglieri CR,

Peerf reviewing PDF | (2021:02:57682:1:2:NEW 13 Apr 2021) 
728 Chacon-Mikahil MPT. Cardiovascular Responses to Different Resistance Exercise Protocols in

729 Elderly. Int J Sports Med 2017; 38:928-36.

730 53. Yasuda T, Fukumura K, Tomaru T, Nakajima T. Thigh muscle size and vascular function

731 after blood flow-restricted elastic band training in older women. Oncotarget 2016; 7:595-607.

732 54. Ramis TR, Muller CH de L, Boeno FP, Teixeira BC, Rech A, Pompermayer MG, Medeiros

733 NS, Oliveira AR, Pinto RS, Ribeiro JL. Effects of Traditional and Vascular Restricted Strength

734 Training Program With Equalized Volume on Isometric and Dynamic Strength, Muscle

735 Thickness, Electromyographic Activity, and Endothelial Function Adaptations in Young Adults.

736 J Strength Cond Res 2020; 34:689-98.

737 55. Kambic T, Novakovic M, Tomazin K, Strojnik V, Jug B. Blood flow restriction resistance

738 exercise improves muscle strength and hemodynamics, but not vascular function in coronary

739 artery disease patients: A pilot randomized controlled trial. Front Physiol 2019; 10:1-11.

740 56. Lixandrão ME, Ugrinowitsch C, Berton R, Vechin FC, Conceição MS, Damas F, Libardi

741 CA, Roschel H. Magnitude of Muscle Strength and Mass Adaptations Between High-Load

742 Resistance Training Versus Low-Load Resistance Training Associated with Blood-Flow

743 Restriction: A Systematic Review and Meta-Analysis. Sport Med 2018; 48:361-78.

744 57. Silva JCG, Pereira Neto EA, Pfeiffer PAS, Neto GR, Rodrigues AS, Bemben MG, Patterson

745 SD, Batista GR, Cirilo-Sousa MS. Acute and Chronic Responses of Aerobic Exercise With

746 Blood Flow Restriction: A Systematic Review. Front Physiol 2019; 10:1-16.

747 58. Spitz RW, Wong V, Bell ZW, Viana RB, Chatakondi RN, Abe T, Loenneke JP. Blood Flow

748 Restricted Exercise and Discomfort. J Strength Cond Res 2020;1.

749 59. Cezar MA, De Sá CA, Da Silva Corralo V, Copatti SL, Dos Santos GAG, Da Silva

750 Grigoletto ME. Effects of exercise training on blood pressure in medicated hypertensive patients

751 with blood flow restriction. Motriz Rev Educ Fis 2016; 22:9-17.

752 60. Araújo JP, Silva ED, Silva JCG, Souza TSP, Lima EO, Guerra I, Sousa MSC. The acute

753 effect of resistance exercise with blood flow restriction with hemodynamic variables on

754 hypertensive subjects. J Hum Kinet 2014; 43:79-85.

755 61. Button KS, Ioannidis JPA, Mokrysz C, Nosek BA, Flint J, Robinson ESJ, Munafo MR.

756 Power failure: Why small sample size undermines the reliability of neuroscience. Nat Rev

757 Neurosci 2013; 14:365-76.

758 62. Turner RM, Bird SM, Higgins JPT. The Impact of Study Size on Meta-analyses:

759 Examination of Underpowered Studies in Cochrane Reviews. PLoS One 2013; 8:1-8.

760 63. Schoenfeld BJ. Potential Mechanisms for a Role of Metabolic Stress in Hypertrophic

761 Adaptations to Resistance Training. Sport Med 2013; 43:179-94.

762 64. Schoenfeld BJ, Ogborn D, Krieger JW. Dose-response relationship between weekly

763 resistance training volume and increases in muscle mass: A systematic review and meta-analysis.

764 J Sports Sci 2017; 35:1073-82.

765 65. Calle MC, Fernandez ML. Effects of resistance training on the inflammatory response. Nutr

766 Res Pract 2010; 4:259.

767 66. Loenneke JP, Fahs CA, Rossow LM, Sherk VD, Thiebaud RS, Abe T, Bemben DA, Bemben

768 MG. Effects of cuff width on arterial occlusion: Implications for blood flow restricted exercise.

769 Eur J Appl Physiol 2012; 112:2903-12.

770 67. Jessee MB, Dankel SJ, Buckner SL, Mouser JG, Mattocks KT, Loenneke JP. The

771 Cardiovascular and Perceptual Response to Very Low Load Blood Flow Restricted Exercise. Int

772 J Sports Med 2017; 38:597-603.

773 68. Graus F, Nunes F, Cavalcante De Souza Leão G, Exel AL, Celeste M, Diniz C. Ankle 
774 Brachial Index in Patients at High Cardiovascular Risk. Brazilian J Cardiol 2012; 25:94-101. 775 69. Patterson SD, Brandner CR. The role of blood flow restriction training for applied 776 practitioners: A questionnaire-based survey. J Sports Sci 2017; 36:123-30.

777 


\section{Table $\mathbf{1}$ (on next page)}

Summary of vascular function outcomes assessed in the studies and differences between BFR and non-BFR conditions

BFR, Blood flow restriction; HI, High intensity; MI, Moderate intensity; LI, Low-intensity; ABI, Ankle-brachial index; PWV, Pulse wave velocity; CAVI, Cardio-ankle vascular index; FMD, Flow-mediated dilatation; RBF, Resting blood flow; RHBF, Reactive hyperemia blood flow ; $\mathrm{RHI}$, Reactive hyperemia index; $\mathrm{TpO}_{2}$, Tissue oxygenation; vWF, von Willebrand factor; $\mathrm{VEGF}_{1}$, Vascular endothelial growth factor; NO, Nitric oxide; GH, Growth hormone, FDP, Fibrin degradation products; SOD, Superoxide dismutase; CAT, Catalase; $=$, No statistically significant difference between conditions with and without BFR; $(+), B F R$ condition promoted significant positive changes when compared to non-BFR; (-), BFR condition promoted significant negative changes when compared to non-BFR. 


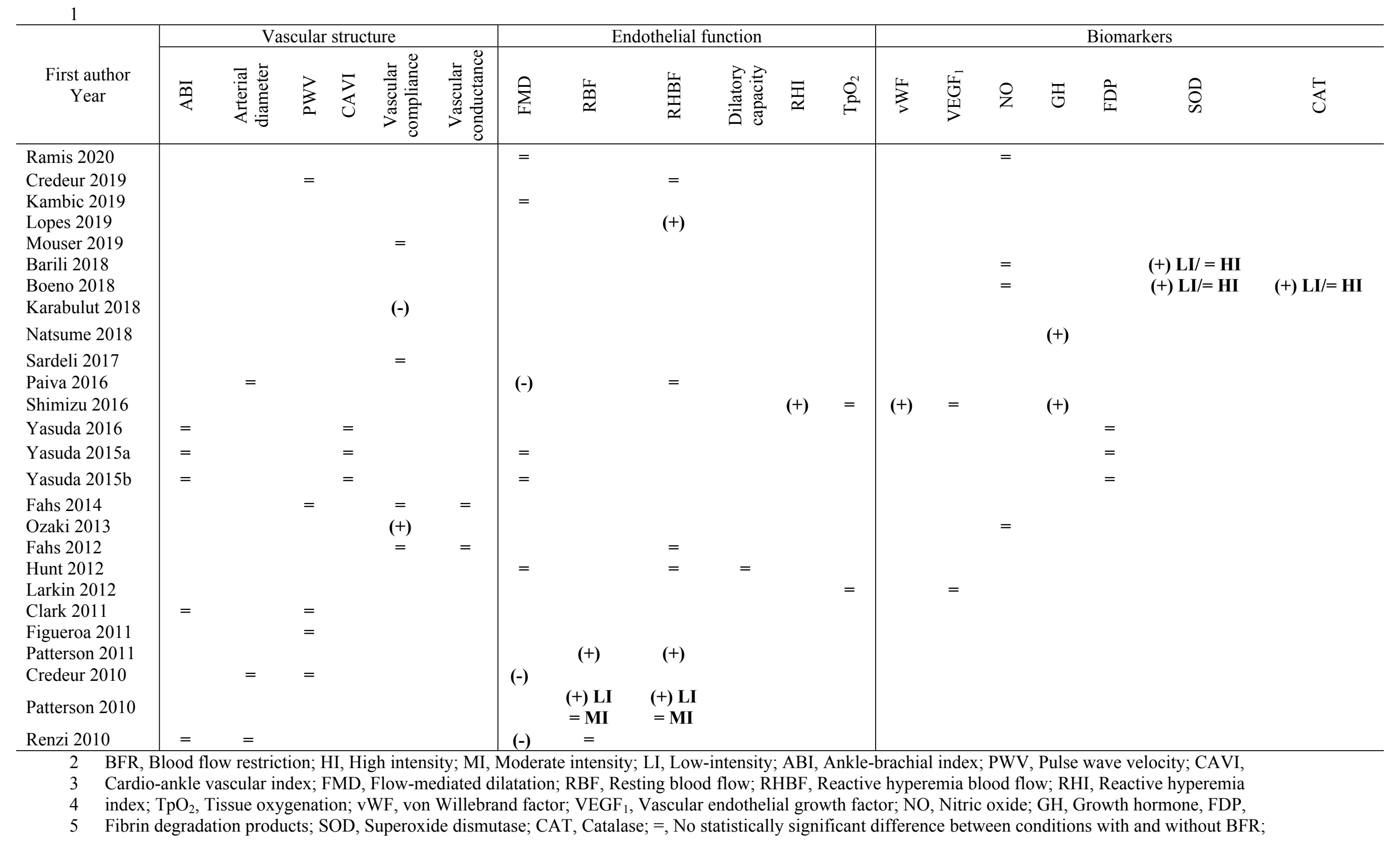


$6 \quad+$ (+), BFR condition promoted significant positive changes when compared to non-BFR; (-), BFR condition promoted significant negative changes when 7 compared to non-BFR. 


\section{Figure 1}

Forest plot presenting comparison of vascular structure between high intensity non-BFR training and low intensity BFRE.

*Values for PWV were reversed to meet direction of vascular compliance where increase means improvement; Squares represent individual study Hedges' g with size corresponding to weight of study and lines are $95 \%$ confidence intervals $(\mathrm{Cl})$; Diamond indicates overall Hedges' g with its width corresponding to $95 \% \mathrm{Cl}$.

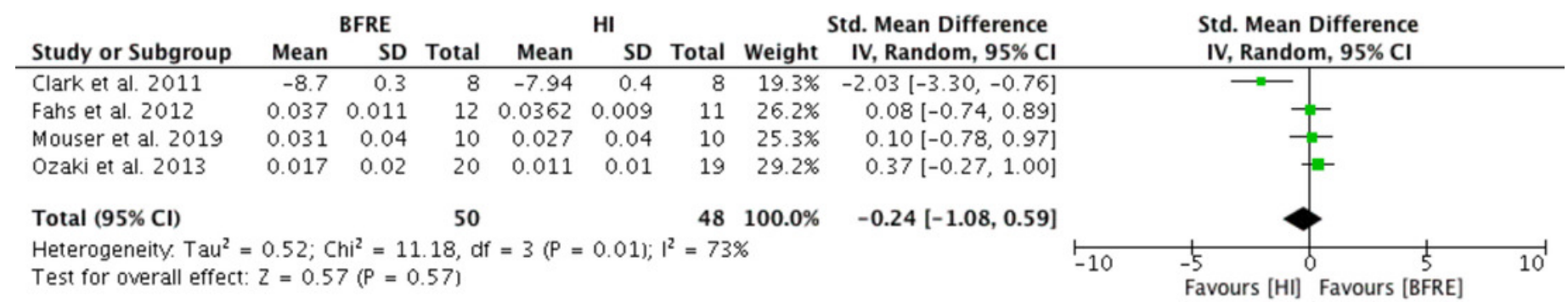


Figure 2

Forest plot presenting comparison of endothelial function between low intensity nonBFR training and low intensity BFRE.

*Increase means improvement for all outcomes; Squares represent individual study Hedges' $\mathrm{g}$ with size corresponding to weight of study and lines are $95 \%$ confidence intervals $(\mathrm{Cl})$; Diamond indicates overall Hedges' g with its width corresponding to $95 \% \mathrm{Cl}$.

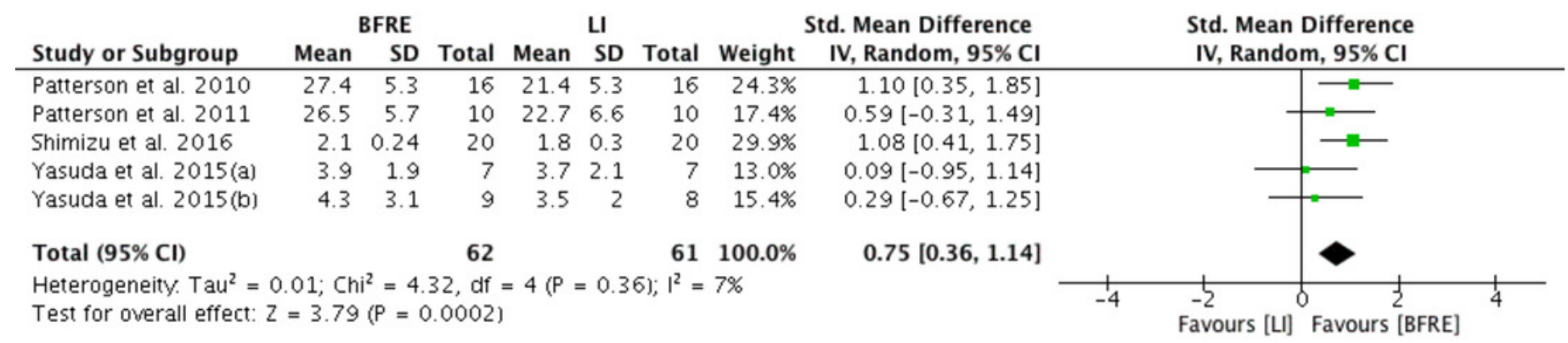

